\title{
Risikoeinschätzung bei ambulant erworbener Pneumonie
}

\section{Wer muss ins Krankenhaus, wer kann zu Hause versorgt werden?}

\begin{abstract}
Um die Gefährdung eines Patienten mit ambulant erworbener Pneumonie zu erkennen, benötigen Sie in der Regel keine aufwendige Diagnostik. Mit vier einfachen, am Krankenbett zu erhebenden Parametern lässt sich das Risiko erfassen und damit das weitere Vorgehen bestimmen.
\end{abstract}

— Der erste Schritt zu einer rationalen Pneumonietherapie ist das Erkennen von Patienten mit Risiken, betonte Prof. Dr. Norbert Suttorp von der Berliner Charité. Ob jemand eine Lungenentzündung problemlos übersteht oder ein lebensbedrohliches Krankheitsbild entwickelt, hängt dabei von zahlreichen Faktoren wie der Virulenz der Erreger, der körpereigenen Abwehr und auch einer genetischen Prädisposition ab.

Ganz wehrlos steht der Körper der Pneumonie nicht gegenüber, erinnerte der Pneumologie. Auch vor der Antibiotikaära überlebten $70 \%$ der Patienten. Über die dahinterstehenden Mechanismen weiß man aber bis heute leider sehr wenig. Genetische Faktoren scheinen keine unwesentliche Rolle zu spielen. Ist ein Elternteil vor dem 50. Lebensjahr an einer Infektion verstorben, ist das Risiko für eine tödliche Infektion fast um den Faktor sechs erhöht.

\section{Mehr als ein Punkt:}

\section{unbedingt stationär behandeln}

Hat man in der Praxis einen Patienten mit Pneumonie vor sich, bringen einen solche Überlegungen nicht weiter. Hier hat sich mit dem CRB-65 ein einfacher klinischer Score bewährt, der ohne Laboruntersuchungen in einer Minute eine Risikoeinschätzung und damit eine Entscheidung über eine Krankenhauseinweisung ermöglicht.

$\mathrm{Ab}$ einem Punkt sollte man bereits eine Krankenhauseinweisung in Erwägung ziehen, sagte Suttorp. Bei zwei und mehr Punkten müsse auf jeden Fall eine Einweisung erfolgen. Die klinische Einschätzung komme bei der

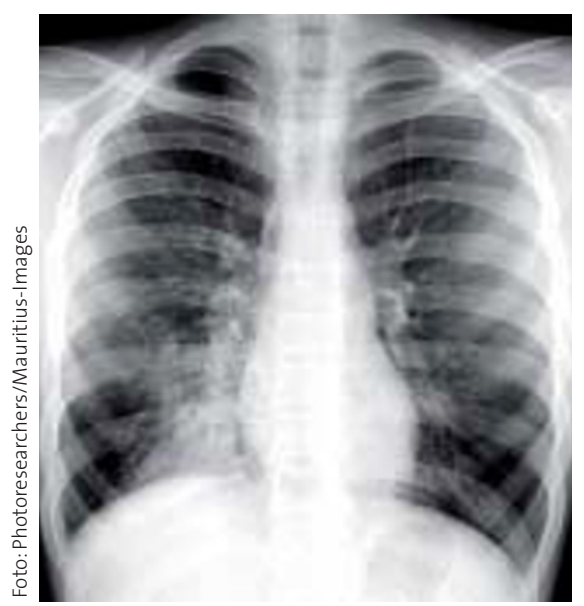

Für die sichere Diagnose einer Pneumonie ist der Nachweis eines Infiltrats in der Röntgen-Thoraxaufnahme erforderlich.

\section{Risikoscore}

\section{CRB-65}

C Confusion

(Bewusstseinstrübung) 1 Punkt

R Respiratory Rate

(Atemfrequenz $\geq 30 / \mathrm{min}$ ) 1 Punkt

B Blood Pressure

(RR diastol. <60 mmHg/

systol. $<90 \mathrm{mmHg}$ ) 1 Punkt

65 Alter $>65$ 1 Punkt

Bewertung der Punkte natürlich immer dazu. So würde man einen Punkt allein aufgrund des Alters natürlich anders bewerten als einen Punkt aufgrund einer Bewusstseinstrübung bei einem 30-Jährigen.

\section{Falsche Verteilung}

In der Realität bestehe hier noch ein Verteilungsproblem, sagte der Pneu- mologe. Etwa 25\% der Pneumoniepatienten im Krankenhaus hätten null Punkte und könnten damit eigentlich ambulant behandelt werden. Auf der anderen Seite werden 33\% der Patienten mit einem Punkt und sogar 5\% der Patienten mit zwei Punkten zu Hause versorgt.

Als zusätzlicher Laborparameter hilft am ehesten Procalcitonin (PCT) bei der Einschätzung des Schweregrades einer Pneumonie weiter. Ein erhöhter Wert deutet auf ein höheres Sterberisiko hin. Ist das PCT niedrig und der CRB-65 liefert drei bis vier Punkte, ist die Diagnose einer Pneumonie fraglich und es liegen der Symptomatik möglicherweise andere Lungenerkrankungen wie Atelektasen zugrunde. Leukozytenzahl und C-reaktives Protein tragen dagegen nur sehr wenig zur Prognoseeinschätzung bei.

\section{Amoxicillin immer noch erste Wahl}

Da die meisten ambulant erworbenen Pneumonien durch Pneumokokken hervorgerufen werden und die Penicillinresistenz in Deutschland sehr niedrig ist, stellt Amoxicillin (3 x $1 \mathrm{~g} / \mathrm{d}$ ) bei leichter unkomplizierter Pneumonie nach wie vor das Mittel der ersten Wahl dar, betonte Suttorp. Makrolide sollten nur bei Penicillinallergie zum Einsatz kommen, da die Resistenzrate hier bis zu 30\% beträgt. In der Praxis werden bei unkomplizierter, ambulant erworbener Pneumonie viel zu häufig Fluorochinolone verschrieben, kritisierte der Pneumologe.

Bei schweren und dann meist auch stationär behandelten Pneumonien sollte von Anfang an mit einer Kombination von Beta-Lactamantibiotika oder Cephalosporinen mit Makroliden behandelt werden.

MARIA WEIß .

- Quelle: Symposium der Paul-Martini-Stiftung, 9./10. November 2007, Berlin 